\title{
Solos subtropicais de altitude: atributos químicos, teor de matéria orgânica e resistência à oxidação química
}

\author{
Highland subtropical soils: chemical attributes, content of organic matter and its resistance \\ to chemical oxidation
}

Letícia Barros da Silva ${ }^{\mathrm{I}}$ Deborah Pinheiro Dick ${ }^{\mathrm{II}}$ Alberto Vasconcellos Inda Junior ${ }^{\mathrm{II}}$

\begin{abstract}
Neste trabalho foram estudadas amostras de RESUMO horizontes A de oito solos representativos da região Campos de Cima da Serra, Rio Grande do Sul, sendo determinados: textura, $\mathrm{pH}$ em água, CTC a $\mathrm{pH} 7$, teores de Al trocável e de óxidos de Fe (Fed e Feo). As amostras foram tratadas com peróxido de hidrogênio $e$, antes e após tratamento, foram determinados teores de $C$ e de $N$ e realizada análise por espectroscopia de infra-vermelho com transformada de Fourier (FTIR). O teor de C foi alto (4 a 23\%) e sua variação correlacionou-se com a altitude e com o teor de Al trocável, sugerindo que a menor temperatura e a complexação com Al iônico contribuem para a estabilização da matéria orgânica do solo (MOS). A proporção de carbono do solo resistente à oxidação variou de 1 a 16\% e correlacionou-se com a razão Feo/Fed. A estabilização desta fração da MOS foi atribuída à interação de grupos carboxílicos ligados a estruturas alifáticas e aromáticas com óxidos de Fe de baixa cristalinidade.
\end{abstract}

Palavras-chave: estabilização, complexação com Al, interação organo-mineral, óxidos de Fe.

\section{ABSTRACT}

In this research, texture, soil $p H, C E C$ at $p H 7$, exchangeable $\mathrm{Al}$, and content of $\mathrm{Fe}$-oxides (Fed and Feo) were determined in A horizons samples of eight representative soils under native pasture from Campos de Cima da Serra, Rio Grande do Sul State, Brazil. The samples were treated with hydrogen peroxide and, before and after the treatment, $C$ and $N$ contents were determined and Fourier Transformed Infrared (FTIR) spectroscopy was performed. The content of soil $C$ was high (4 to 23\%) and its variation correlated with the altitude and exchangeable $\mathrm{Al}$ indicating that lower temperatures and complexation with ionic Al contribute to soil organic matter
(SOM) stabilization. The proportion of oxidation-resistant carbon varied between 1 and $16 \%$ and correlated with the ratio Feo/Fed. The stabilization of this SOM fraction was assigned to the interaction of carboxylic groups bound to aromatic and aliphatic structures with Fe-oxides of low crystallinity degree.

Key words: stabilization, Al complexation, organo-mineral interaction, $\mathrm{Fe}$-oxides.

Na região fisiográfica dos Campos de Cima da Serra, localizada no nordeste do Estado do Rio Grande do Sul, a elevada precipitação pluviométrica e as baixas temperaturas propiciam o acúmulo de matéria orgânica do solo (MOS) nos horizontes superficiais (STRECK et al., 2002). O efeito da textura no teor de MOS se verifica principalmente na formação de complexos organo-minerais, sendo que solos mais argilosos tendem a apresentar maiores teores de C do que solos de textura mais grosseira (HASSINK, 1997). A formação do complexo organo-mineral e sua estabilidade é também afetada pela especificidade da reação, destacando-se os óxidos de Fe e de $\mathrm{Al}$ como os sorventes mais importantes no solo (DICK et al., 2003). A interação da MOS com íons metálicos pode contribuir para sua estabilização química e os íons metálicos considerados como potenciais estabilizantes da MOS são $\mathrm{Ca}^{+2}, \mathrm{Al}^{+3} \mathrm{e} \mathrm{Fe}^{+3}$, além de metais pesados (LÜTZOW et al., 2006). Considerando-se que estudos sobre os

'Programa de Pós-graduação em Ciência do Solo (PPGCS), Universidade Federal do Rio Grande do Sul (UFRGS), Porto Alegre, RS, Brasil.

"Departamento de Físico-Química, Instituto de Química, UFRGS, 91501-970, Porto Alegre, RS, Brasil. E-mail: dpdick@iq.ufrgs.br. Autor para correspondência.

IIIDepartamento de Solos, Faculdade de Agronomia, UFRGS, Porto Alegre, RS, Brasil. 
solos da região são incipientes, este trabalho se propôs a determinar os atributos químicos e quantificar e caracterizar a MOS de classes de solos representativos da região, com ênfase em identificar fatores que afetam sua estabilização em condições nativas.

Para tanto, foram coletadas amostras de 0 a $10 \mathrm{~cm}$ de profundidade no horizonte A de 8 solos sob campo nativo da região de Campos de Cima da Serra, Rio Grande do Sul $\left(28^{\circ}\right.$ a $29^{\circ} \mathrm{S}, 50^{\circ}$ a $52^{\circ} \mathrm{W}$ ) (Tabela 1$)$. O clima da região é temperado (Cfb 1), com temperatura média anual de $14,4^{\circ} \mathrm{C}$, e precipitação média anual de $2.468 \mathrm{~mm}$ ano $^{-1}$, com exceção da região da amostra de Latossolo $\left(17,0^{\circ} \mathrm{C}\right.$ e $\left.1.800 \mathrm{~mm}^{2} \mathrm{ano}^{-1}\right)$. Na fração $<2 \mathrm{~mm}$, foram determinados os atributos químicos do solo (TEDESCO et al., 1995) e os teores de óxidos de Fe pedogênicos (Fed) (MEHRA \& JACKSON, 1960) e de baixa cristalinidade (Feo) (SCHWERTMANN, 1964). A razão Feo/Fed foi calculada.

A massa de $1 \mathrm{~g}$ de solo foi oxidada com $30 \mathrm{~mL}$ de $\mathrm{H}_{2} \mathrm{O}_{2} 30 \%(\mathrm{v} / \mathrm{v})$ sob aquecimento de $40^{\circ} \mathrm{C}$ (EUSTERHUES et al., 2005). Após repetição deste procedimento (2x), a amostra foi lavada com água deionizada $(3 \mathrm{x})$ e seca à $60^{\circ} \mathrm{C}$. Os teores de $\mathrm{C}$ e $\mathrm{N}$ foram determinados por combustão seca (Perkin Elmer 2400) nas amostras de solo ( $\mathrm{C}$ e $\mathrm{N}$ ) e nas tratadas com peróxido $\left(\mathrm{C}_{\mathrm{p}}\right)$. A fração da MOS resistente à oxidação com peróxido foi calculada dividindo $\mathrm{C}_{\mathrm{p}}$ por $\mathrm{C}$ e multiplicando por $100\left(\mathrm{C}_{\mathrm{p}} / \mathrm{C}, \%\right)$. As amostras de solo antes e após oxidação foram analisadas por espectroscopia de infra-vermelho com transformada de Fourier (FTIR) por transmissão (Shimadzu FTIR 8300), segundo DICK et al. (2003), e a interpretação dos espectros, segundo TAN $(1996,2003)$. Foram realizadas correlações lineares e a significância do coeficiente de correlação $r$ foi avaliada pelo programa MultivMinorWindons v.2.3.21 (PILLAR, 2004) via teste de aleatorização, considerando como limiar á=0,01.

Com exceção do Gleissolo GH6, a textura dos solos variou de argilosa a muito argilosa (Tabela 1). Os solos são fortemente ácidos $(4,3 \leq \mathrm{pH} \leq 4,8)$ e apresentam média a alta CTC a pH 7 (13,0 $\leq$ CTC $\leq$ 25,6 cmol $\mathrm{kg}^{-1}$ ). Em geral, o teor de Al trocável e a saturação de Al são altos (Tabela 1). No LB12, que apresenta estágio mais avançado de intemperismo, os valores para ambos atributos são inferiores. Os teores de Feo $\left(0,9 \leq\right.$ Feo $\left.\leq 10,9 \mathrm{~g} \mathrm{~kg}^{-1}\right)$ e de Fed $(3,6 \leq$ Fed $\leq$ $59,9 \mathrm{~g} \mathrm{~kg}^{-1}$ ) variaram amplamente. Os elevados valores da razão Feo/Fed indicam que parte considerável dos óxidos de Fe pedogênicos encontra-se em formas de baixa cristalinidade (ALMEIDA et al., 1997) (Tabela 1).

$\mathrm{O}$ teor de $\mathrm{C}$ dos solos foi alto e o teor de $\mathrm{N}$ oscilou entre 0,23 e 1,4 \% (Tabela 1). Entre as correlações

Tabela 1 - Classificação dos solos, código da amostra, altitude, teor de argila, pH em água, CTC a pH 7, teor de alumínio trocável, saturação por alumínio, teores de carbono e de nitrogênio do solo ( $\mathrm{C}$ e $\mathrm{N}$ ), razão $\mathrm{C} / \mathrm{N}$ do solo, teores de óxidos de Ferro de baixa cristalinidade (Feo) e pedogênicos (Fed) do solo, razão Feo/Fed e proporção do carbono do solo resistente à oxidação (Cp/C).

\begin{tabular}{|c|c|c|c|c|c|c|c|}
\hline Solo Classificação & Código & Altitude & argila & $\mathrm{pH}_{\mathrm{H} 2 \mathrm{O}}$ & $\mathrm{CTC}_{\mathrm{pH}} 7$ & $\mathrm{Al}_{\text {trocável }}$ & $\mathrm{Al}_{\text {Sat. }}$ \\
\hline & & $\mathrm{m}$ & $\mathrm{g} \mathrm{kg}^{-1}$ & & \multicolumn{2}{|c|}{----- $\mathrm{cmol}_{\mathrm{C}} \mathrm{kg}^{-1}$----- } & ---\%-- \\
\hline Neossolo Litólico* & NL2 & 923 & 420 & 4,7 & 19,8 & 5,6 & 28,3 \\
\hline Gleissolo Melânico* & GM4 & 1014 & 560 & 4,3 & 25,6 & 11,7 & 45,7 \\
\hline Gleissolo Háplico* & GH6 & 986 & 320 & 4,7 & 13,0 & 4,0 & 30,8 \\
\hline Neossolo Litólico* & NL7 & 1215 & 570 & 4,6 & 17,4 & 8,2 & 47,2 \\
\hline Cambissolo Hístico* & CHi8 & 1108 & 500 & 4,6 & 17,7 & 7,4 & 41,8 \\
\hline Cambissolo Húmico** & CHu9 & 980 & 600 & 4,5 & 16,5 & 5,3 & 32,1 \\
\hline Cambissolo Húmico** & CHu10 & 939 & 540 & 4,5 & 18,4 & 6,3 & 34,2 \\
\hline Latossolo Bruno*** & LB12 & 909 & 610 & 4,8 & 16,2 & 2,9 & 17,9 \\
\hline \multirow[t]{2}{*}{ Solo/Classificação } & $\mathrm{C}$ & $\mathrm{N}$ & $\mathrm{C} / \mathrm{N}$ & Feo & Fed & Feo/Fed & $\mathrm{C}_{\mathrm{P}} / \mathrm{C}$ \\
\hline & \multicolumn{2}{|c|}{-----------\%"--------- } & & \multicolumn{2}{|c|}{---------g kg k'--------- } & & $\%$ \\
\hline Neossolo Litólico* & 6,3 & 0,42 & 15 & 4,6 & 45,3 & 0,10 & 4,1 \\
\hline Gleissolo Melânico* & 23,3 & 1,42 & 16 & 0,9 & 3,6 & 0,25 & 15,6 \\
\hline Gleissolo Háplico* & 5,1 & 0,40 & 13 & 2,8 & 14,5 & 0,19 & 10,6 \\
\hline Neossolo Litólico* & 12,9 & 0,77 & 17 & 10,9 & 37,1 & 0,29 & 9,8 \\
\hline Cambissolo Hístico* & 8,4 & 0,52 & 16 & 6,8 & 38,6 & 0,18 & 9,2 \\
\hline Cambissolo Húmico** & 4,1 & 0,30 & 14 & 7,2 & 54,6 & 0,13 & 13,0 \\
\hline Cambissolo Húmico** & 4,6 & 0,23 & 20 & 3,9 & 41,1 & 0,10 & 5,4 \\
\hline Latossolo Bruno*** & 4,5 & 0,41 & 11 & 6,7 & 59,9 & 0,11 & 1,0 \\
\hline
\end{tabular}

Locais de coleta no Estado do Rio Grande do Sul: São José dos Ausentes(*); Bom Jesus(**);Vacaria (***). 
testadas com teor de $\mathrm{C}$, apenas as realizadas com o $\mathrm{Al}$ trocável e com a altitude foram estatisticamente significativas em nível de $1 \%(r=0.915$ e $r=0,919$, respectivamente). No entanto, o melhor ajuste dos dados para teor de $\mathrm{C}$ e $\mathrm{Al}$ trocável corresponde a uma equação polinomial $\left(\mathrm{r}^{2}=0,964, \mathrm{P}=2,54 \cdot 10^{-4}\right)$, que indica que a proteção química da MOS devido à complexação com $\mathrm{Al}$ é relevante para teores de $\mathrm{Al}$ trocável maiores do que $5,5 \mathrm{cmol}_{\mathrm{c}} \mathrm{kg}^{-1}$. Resultados semelhantes foram obtidos por SCHWESIG et al. (2003). Na correlação entre o teor de $\mathrm{C}$ e a altitude, foi excluído o Gleissolo GM4, uma vez este apresenta um horizonte hístico com alto teor de $\mathrm{C}$ e com um regime hídrico diferenciado dos demais solos, em que a dinâmica da MOS é afetada principalmente pelo ambiente anaeróbico periódico. Quanto às demais amostras, este resultado indica que mesmo em solos de elevada altitude (>900 m) o aumento da mesma propicia um acúmulo de MOS, devido à diminuição da temperatura e ao aumento da saturação do solo, os quais diminuem a taxa de decomposição dos resíduos pelos microorganismos (BAYER \& MIELNICZUK, 1999). O fato de o teor de C não apresentar correlação com o teor de Fed e com o teor de argila indica que os óxidos de Fe e os minerais da fração argila não afetam relevantemente a estabilização da MOS total nestes solos de altitude, em oposição ao observado para Latossolos brasileiros com menor teor de C no horizonte A(DICK et al., 2005).

A razão C/N variou de 13 a 20 (Tabela 1 ) nos Neossolos, Gleissolos e Cambissolos independentemente da classe de solo, da altitude e do teor de Al trocável. No Latossolo esta razão foi a menor do grupo (Tabela 1), o que sugere que neste solo a MOS apresenta comparativamente um maior grau de humificação (DICK et al. ,2005).

Os espectros de FTIR dos solos com $\mathrm{Ct} \leq 8,4 \%$ foram similares ao da amostra NL2 (Figura 1a). Em todos os espectros foram observadas bandas de absorção em 3697 e $3622 \mathrm{~cm}^{-1}$, atribuídas às vibrações $\mathrm{OH}$ da ligação Al-OH, além de uma banda em $1084 \mathrm{~cm}^{-1}$ referente às vibrações O-Al-OH. Estas bandas indicam a presença de caulinita, que é confirmada pela vibração de O-Al-OH em 1084 e $912 \mathrm{~cm}^{-1}$ e pela vibração de Si-O em $1009 \mathrm{~cm}^{-1}$. A banda em $1635 \mathrm{~cm}^{-1}$ é usualmente atribuída à presença de água estrutural e a banda em $1034 \mathrm{~cm}^{-1}$ é atribuída à ligação Si-O de quartzo. Um triplete entre 3600 e $3400 \mathrm{~cm}^{-1}$, observado no espectro da amostra NL7 (3527, 3447 e 3390 cm-1 $)$, típico da vibração O-Al-OH da gibbsita, indica a presença deste mineral neste solo. No espectro da amostra GM4, as bandas da caulinita e da gibbsita foram menos pronunciadas devido ao alto teor de MOS. Neste solo as bandas de absorção devido à MOS foram comparativamente melhor definidas, destacando-se: $3438 \mathrm{~cm}^{-1}$ (estiramento de O-H em sobreposição à banda de gibbsita), 2922 e $2855 \mathrm{~cm}^{-1}$ (estiramento $\mathrm{C}-\mathrm{H}$ de grupos alifáticos), $1728 \mathrm{~cm}^{-1}$ (estiramento de $\mathrm{C}=\mathrm{O}$ de $\mathrm{COOH}$ ), dubleto em 1635 e $1390 \mathrm{~cm}-1$ (estiramento $\mathrm{C}=\mathrm{O}$ de $\mathrm{COO}^{-}$, em sobreposição à de $\mathrm{H}_{2} \mathrm{O}$ estrutural). Esta composição, semelhante em todos os solos analisados, é típica de matéria orgânica humificada.

$\mathrm{O}$ teor de $\mathrm{C}$ remanescente após oxidação com $\mathrm{H}_{2} \mathrm{O}_{2}\left(\mathrm{C}_{\mathrm{p}}\right)$ variou de 0,23 a 3,57\%, correspondendo de 4 a $16 \%$ do carbono total (razão $\mathrm{C}_{\mathrm{P} / \mathrm{C}}$ ) (Tabela 1). O teor de $\mathrm{C}_{\mathrm{p}}$ correlacionou-se apenas com a razão $\mathrm{Feo} /$ Fed $(\mathrm{r}=0,968, \mathrm{P}<0,01)$, indicando que parte da MOS é estabilizada pela interação com a fração de óxidos de Fe de baixa cristalinidade. Comportamento semelhante foi observado por EUSTERHUES et al. (2005). No solo GM4, excluído da correlação, provavelmente a MOS não tenha sido eficientemente acessada pelo peróxido devido ao seu elevado teor. Os espectros de FTIR de três amostras tratadas com $\mathrm{H}_{2} \mathrm{O}_{2}$ (Figura 1b) apresentam, em comparação com os solos não-tratados (Figura 1a) uma diminuição considerável da intensidade da banda em 3438 $\mathrm{cm}^{-1}$ (para NL2) e das bandas em 2927 e $1635 \mathrm{~cm}^{-1}$, e desaparecimento da banda em $2855 \mathrm{~cm}^{-1}$. Em contrapartida, a banda em $3128 \mathrm{~cm}^{-1}$ do estiramento C$\mathrm{H}$ de grupos aromáticos apresenta melhor definição, sendo ainda observados: estiramento $\mathrm{C}=\mathrm{O}$ do grupo carboxílico em $1721 \mathrm{~cm}^{-1}$, vibração $\mathrm{C}=\mathrm{C}$ de grupos aromáticos em $1696 \mathrm{~cm}^{-1}$ sobreposta ao estiramento simétrico de $\mathrm{COO}^{-}$, estiramento assimétrico de $\mathrm{COO}^{-}$ em $1400 \mathrm{~cm}^{-1}$, e estiramento C-O de ácido carboxílico em $1291 \mathrm{~cm}^{-1}$ (Figura 1b). Portanto, a MOS resistente à oxidação é composta por grupos carboxilato e carboxílicos associados a estruturas alifáticas e aromáticas, conforme já observado por EUSTERHUES et al. (2005) em MOS de solos de clima temperado.

A variação do teor de $\mathrm{C}$ de horizonte $\mathrm{A}$ dos solos de altitude estudados independe da classe de solo, da textura e do teor de óxidos de Ferro pedogênicos. A complexação com $\mathrm{Al}^{+3}$ trocável estabiliza a matéria orgânica do horizonte A. A diminuição da temperatura e o aumento da saturação do solo, expressos pelo aumento da altitude, também contribuem para o acúmulo de $\mathrm{C}$ neste ambiente de altitude. A importância da interação organo-mineral na estabilização da MOS se verifica em até $15 \%$ do teor original da mesma.

\section{AGRADECIMENTOS E APRESENTAÇÃO}

À Fundação de Amparo à Pesquisa do Estado do Rio Grande do Sul (FAPERGS) e Coordenação de 


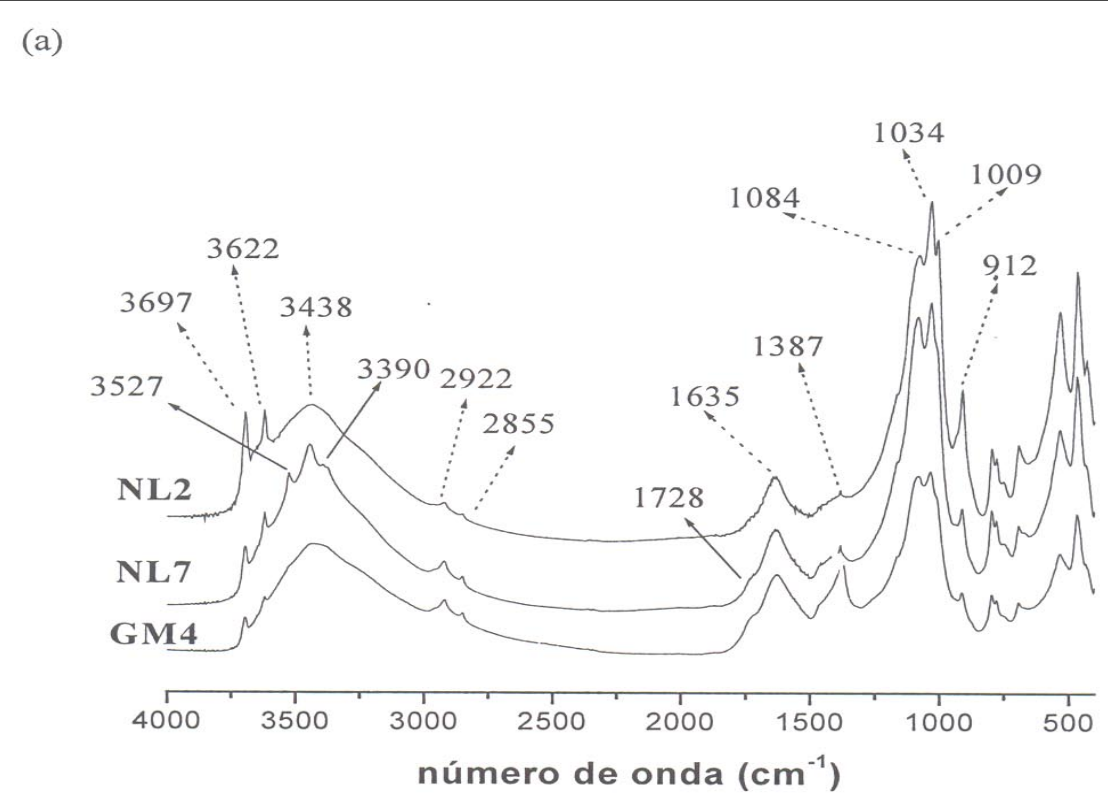

(b)

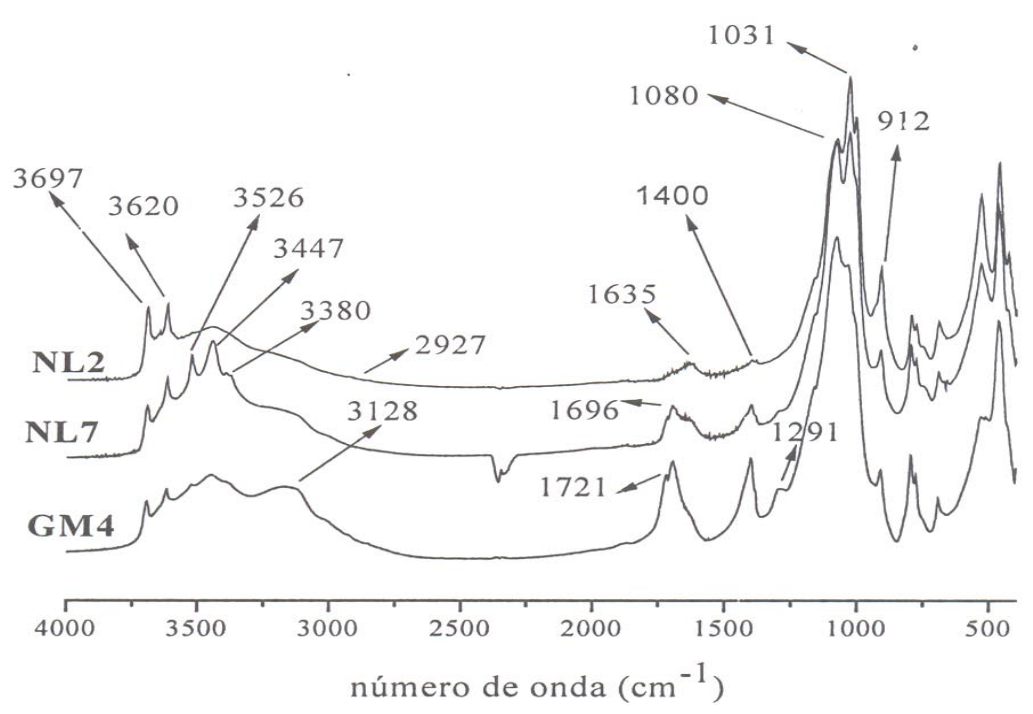

Figura 1 - Espectros de FTIR dos Neossolos NL2 e NL7 e do Gleissolo GM4 antes (a) e após o tratamento com $\mathrm{H}_{2} \mathrm{O}_{2}$ (b).

Aperfeiçoamento de Pessoal de Nível Superior (CAPES) e ao DAAD pelo financiamento do projeto e ao Conselho Nacional de Desenvolvimento Científico e Tecnológico (CNPq), pelas bolsas de produtividade em pesquisa e de mestrado.

Parte da dissertação apresentada pela primeira autora ao Programa de Pós-graduação em Ciências do Solo, Universidade Federal do Rio Grande do Sul (UFRGS), RS, Brasil.

\section{REFERÊNCIAS}

ALMEIDA, J.A. et al. Gênese do contraste textural e da degradação do horizonte B de um Podzólico vermelho-amarelo da Planície costeira do Rio Grande do Sul. Revista Brasileira de Ciência do Solo, v.21, p.221-233, 1997.

BAYER, C.; MIELNICZUK, J. Dinâmica e função da matéria orgânica. In: SANTOS, G.A.; CAMARGO, F.A.O. Fundamentos da matéria orgânica do solo. Porto Alegre: Gênesis, 1999. p.9-26.

DICK, D.P. et al. Chemical characterization and infrared spectroscopy of soil organic matter from two southern Brazilian soils. Revista Brasileira de Ciência do Solo, v.27, p.2939, 2003. 
DICK, D.P. et al. Characteristics of soil organic matter of different Brazilian Ferralsols under native vegetation as a function of soil depth. Geoderma, v.124, p.319-333, 2005.

EUSTERHUES,K. et al. Stabilization of organic matter isolated via oxidative degradation. Organic geochemistry, v.36, p.1567-1575, 2005.

HASSINK, J. The capacity of soils to preserve organic C and N by their association with clay and silt particles. Plant Soil, v.191, p.77-87, 1997.

LÜTZOW, M.V. et al. Stabilization of organic matter in temperate soils: mechanisms and their relevance under different soil conditions- a review. European Journal of Soil Science, v.57, p.426-445, 2006.

MEHRA, O.P.; JACKSON, M.L. Iron oxide removal from soils and clays by a dithionite-citrate system buffered with sodium bicarbonate. Clays Clay Minerals, v.7, p.317-327, 1960.

PILLAR, V.D. MULTIV: Multivariate exploratory analysis, randomization testing and bootstrap resampling. Use's guide. Porto Alegre: Univeridade Federal do Rio Grande do Sul, 2004. V.2.4. 50p.
SCHWERTMANN, U. Differenzierung der eisenoxide des bodens durch extraktion mit ammonium - Oxalat Losung. Zeitschrift fur Pflanzenernährung und Boden Analysis, v.105, p.194-202, 1964.

SCHWESIG, D. et al. Effects of aluminium on the mineralization of dissolved organic carbon derived from forest floors. European Journal of Soil Science, v.54, p.311-322, 2003.

STRECK, E.V. et al. Solos do Rio Grande do Sul. Porto Alegre: UFRGS, 2002. 130p.

TAN, K.H. Infrared spectroscopy. In: TAN, H.K. Soil sampling, preparation and analysis. New York: Marcel Dekker, 1996. p.278-298.

TAN, K.H. Humic matter in soil and the environment. Principles and controversies. New York: Marcel Dekker, 2003. 385p.

TEDESCO, M.J. et al. Análise de solo, plantas e outros materiais. 2.ed. Porto Alegre: Departamento de Solos da UFRGS, 1995. 174p. (Boletim Técnico, 5). 